\title{
QUERIES 'N THEORIES: AN INSTRUCTIONAL GAME ON \\ THE DOT, DOT, DOT, . . . APPROACH TO SCIENTIFIC METHOD
}

\author{
LAYMAN E. ALLEN \\ University of Michigan
}

\begin{abstract}
QUERIES 'N THEORIES provides a parallel to the strong inference approach to scientific method - designing experiments, observing data, and theorizing. The reiterated use of the DOT approach (Design, Observe, Theorize) in the problem-solving required by the game mirrors the regular, systematic application of strong inference in some areas of science (e.g., high energy physics and molecular biology) that have moved ahead much more rapidly than others. Moreover, the game embodies and provides practice in two aspects of scientific theorizing and designing which John Platt has pointed out as central to scientific advance: (1) the usefulness of multiple hypotheses and (2) disproof as science's mode of advance.

Players of QUERIES 'N THEORIES assume the roles of a "native" and of linguists ("querists") who are attempting to understand the native's language in a defined sense. Using strings of colored chips, the native secretly constructs the basic sentences and the replacement rules (if any) of his language. The querists ask questions by constructing queries (strings of colored chips) on the query mat. They seek to achieve an understanding of the language such that when the native in turn asks them about it by constructing queries on the query mat, they will be able to answer correctly. The goal is to ask the fewest number of questions necessary to achieve the specified understanding.

By presenting a programmed series of sample games that gradually and steadily increase in complexity, the author attempts to demonstrate that QUERIES ' $N$ THEORIES offers its players the opportunity to learn a powerful analytic skill. He concludes that the examples are sufficiently persuasive to indicate the value of rigorous empirical investigation of a series of hypotheses about the merits of QUERIES 'N THEORIES as a vehicle for practicing and improving skill in the use of the strong inference approach to scientific method.
\end{abstract}

Observing and theorizing and designing experiments - and doing all three well - are the intellectual keys to scientific progress. In his classic article on scientific method, John Platt has convincingly set forth the case 
for more widespread systematic application of man's most powerful approach to problem solving (Platt, 1964). However, to characterize the crucial processes of scientific research is one thing; to learn how to carefully observe, to imaginatively theorize, and to rigorously design is quite another matter. It is to this learning problem that the instructional game QUERIES 'N THEORIES (Allen et al., 1970) is addressed.

Convinced of the importance of examining the strong inference approach of science to determine whether other groups and individuals might learn to adopt it profitably in their own scientific and intellectual work, Platt observes that some fields of science are moving ahead very much faster than others. He observes further that in such rapidly advancing fields as molecular biology and high energy physics the techniques of strong inference are applied formally and explicitly and regularly and indicates his belief that this systematic application is the primary factor in such advances. Platt characterizes strong inference as follows:

In its separate elements strong inference is just the simple and old-fashioned method of inductive inference that goes back to Francis Bacon. The steps are familiar to every college student and are practiced off and on by every scientist. The difference comes in their systematic application. Strong inference consists of applying the following steps to every problem in science, formally and explicitly and regularly:

(1) Devising alternative hypotheses;

(2) Devising a crucial experiment (or several of them), with alternative possible outcomes, each of which will, as nearly as possible, exclude one or more of the hypotheses;

(3) Carrying out the experiment so as to get a clean result;

(1') Recycling the procedure, making subhypotheses or sequential hypotheses to refine the possibilities that remain; and so on.

An exact parallel to the strong inference which Platt describes above is called the DOT, DOT, DOT, ... approach to problem solving in QUERIES ' $N$ THEORIES. The ' $D$ ' in DOT stands for Design of experiments (Platt's \#2, devising crucial experiments); the ' $O$ ' stands for Observation (Platt's \#3, carrying out the experiment to generate new data for observation); and the ' $T$ ' stands for Theorizing (Platt's \#1, devising alternative hypotheses). Calling it the DOT, DOT, DOT, . . . approach emphasizes that its power derives from the repetitive, regular, systematic application of its subsidiary steps. Or as Platt states it more eloquently:

... [W] do not realize the added power that the regular and explicit use of alternative hypotheses and sharp exclusions could give us at every step of our research. 
The difference between the average scientist's informal methods and the methods of the strong-inference users is somewhat like the difference between a gasoline engine that fires occasionally and one that fires in steady sequence. If our motorboat engines were as erratic as our deliberate intellectual efforts, most of us would not get home for supper.

Platt finds further testimony to the power of strong inference in the achievements of its use in organic chemistry and yet more in the work habits of some of the world's most respected scientists: Faraday, Fermi, Roentgen, Pasteur, Newton, and Maxwell. He emphasizes the theorizing and designing aspects of strong inference, while in QUERIES ' $N$ THEORIES the importance of observing carefully and completely is equally stressed. To illustrate how QUERIES 'N THEORIES exemplifies Platt's pair of central themes about theorizing and designing (namely, (1) the usefulness of multiple hypotheses and (2) disproof as science's basic mode of advance), we turn now to a consideration of this game of science and language.

QUERIES 'N THEORIES is a game in the sense of Von Neumann and Morgenstern's definition (Von Neumann and Morgenstern, 1944) namely, that a game is "the totality of the rules that describe it." Within the limited and strictly defined universe imposed by the rules of any game, players are given opportunities to test and evaluate the results of many varieties of behavior.

In simple games, players are probably learning only how to play better the game in question; a good instructional game is one that makes it possible for players to try out varieties of behavior that may be useful to them in other contexts. One of the goals in designing QUERIES ' $N$ THEORIES was to provide an occasion in which players are confronted in the context of playing the game with a graded series of problems, starting with simple ones and proceeding to ones of increasing complexity. The problems are of a type that furnish an opportunity to practice the use of the strong inference brand of scientific method and to test its usefulness and power in comparison with alternative approaches. Described somewhat more generally, QUERIES 'N THEORIES has been designed to develop a basic and uniquely human skill: asking good questions. In the game, players ask questions by constructing queries. But to construct good queries, a player must be a good theorist - skillful in organizing, analyzing, and synthesizing data. These processes of formulating theories and testing them by constructing queries are deliberately parallel to the methods used in science to probe the laws of Nature.

The hope, of course, is that QUERIES 'N THEORIES is a good instructional game in the sense that the skill and insight achieved from recurrent practice in theorizing and constructing queries in the game will 
prove relevant for enhancing talent in scientific theorizing and experimental design. With the completion and availability of the game, that hypothesized and hoped-for relevance can now be tested

I do not at this time have data to offer from completed experiments that will disconfirm the hypothesis that the hoped-for relevance is absent. What I can do now is to describe in detail what happens in the play of the game so that you can bring your own intuitions to bear in judging the likelihood that the time-compressed experience in designing, observing, and theorizing which occurs in the play will be accompanied by improved performance in the use of scientific method. The question is straightforward and an interesting one: How likely is how much practice with the DOT, DOT, DOT, .... approach to problem-solving in QUERIES ' $N$ THEORIES to be accompanied by how much improvement in strong inference problem-solving in science?

The play of QUERIES 'N THEORIES involves one player's formulating a language and the other players' seeking to achieve an "understanding" of that language in a defined sense by asking questions of the language-builder (in the game he is called the "native") about the language that he has built. The goal is to ask the fewest number of questions necessary to achieve the specified understanding.

What is a language for purposes of QUERIES 'N THEORIES? One can begin to answer the question by giving some examples of languages in QUERIES 'N THEORIES. The following (L1) is a simple finite language that the native might have formulated by placing yellow (Y) and blue (B) chips in appropriate positions on the language mat.

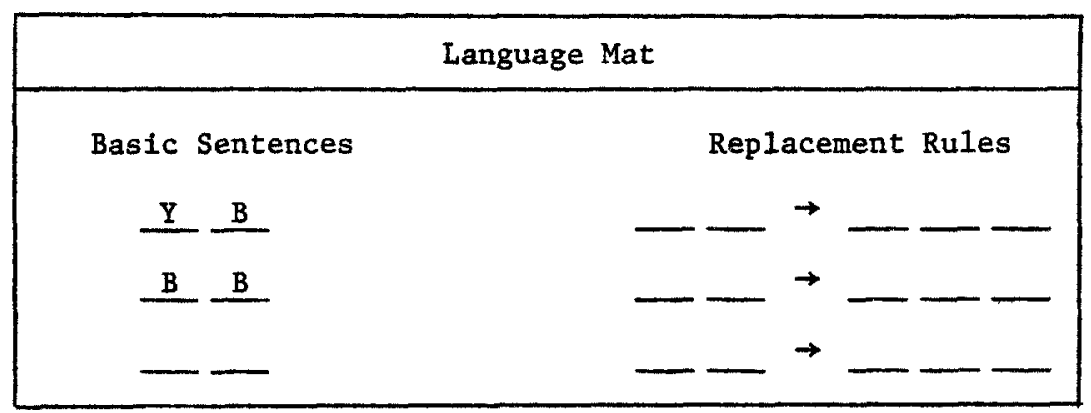

A scientist seeking to understand the native's language (in the game he is called a "querist") is empowered by the game rules to perform certain experiments, the results of which provide data about the native's language. A querist can pose certain questions about the native's language; these the native must answer truthfully. The penalties in the scoring rules are very heavy for "native who speak with forked tongue!" The querist asks questions by constructing queries, that is, by placing colored chips on 
the query mat. The following string of colored chips placed in row 1 would ask the question: "Native, is the string of chips consisting of a yellow chip and a blue chip, in that order, a sentence in your language?"

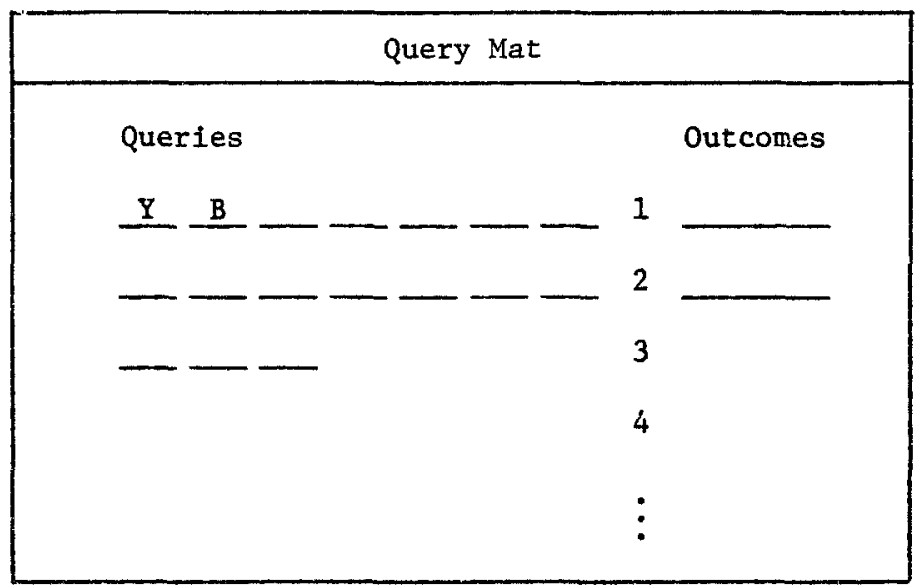

The native would respond by placing a red chip (to indicate No) or a green chip (to indicate Yes) in the outcome column. In the case of language $\mathrm{L} 1$ the native would respond with a green chip.

L1 $\quad$ I B $-1-1$

If called upon to prove that yellow-blue was a sentence in his language, the native could do so by merely pointing to the basic sentences in his language and showing that yellow-blue is included there.

If a querist next asked, "Native, is the string yellow-yellow a sentence in your language?" what should the response be?

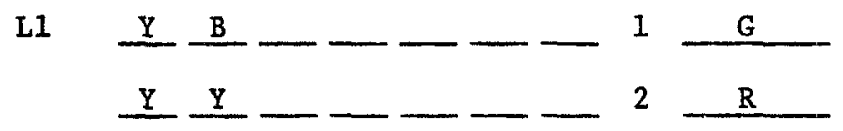

That's right, the response should be red, because yellow-yellow is not a sentence in language L1. Similarly, if the queries blue-blue and blue-yellow were constructed in rows 3 and 4 , the responses by the native should be green and red, respectively.

L1

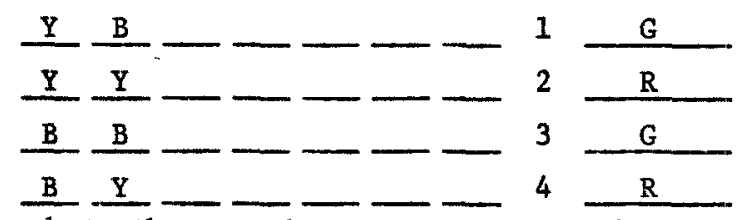

No matter what other queries are constructed for $\mathrm{L} 1$, the responses should be red, because L1 is a finite two-sentence language containing only sentences $\mathrm{YB}$ and $\mathrm{BB}$. Now that we have learned language L1 perfectly, let's consider L2. 


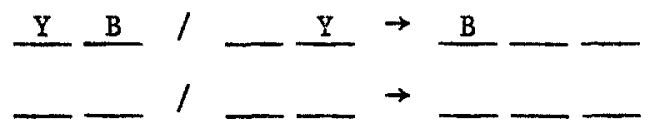

Language L2 has only one basic sentence - namely, yellow-blue. But L2 has something that $\mathrm{L} 1$ does not have: a replacement rule. The single yellow chip to the left of the arrow with the single blue chip to the right of the arrow in the replacement rule in $\mathrm{L} 2$ means that anywhere that a yellow chip occurs in a sentence of L2, that yellow chip may be replaced by a blue chip and the result will be a sentence of $\mathrm{L} 2$. If the same four questions are asked of $\mathrm{L} 2$ as were asked of $\mathrm{L} 1$, the responses should be exactly the same.

L2

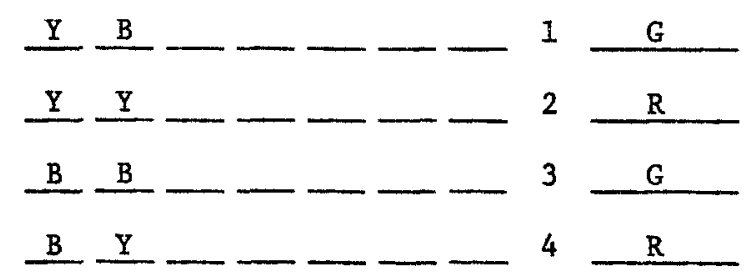

If asked to prove his affirmative responses to $\# 1$ and $\# 3$, the native could do so by indicating: YB is a sentence in $\mathrm{L} 2$, because it is a basic sentence; and $B B$ is a sentence of $L 2$ because it is the result of applying the replacement rule, $Y \rightarrow B$, to the basic sentence, $Y B$.

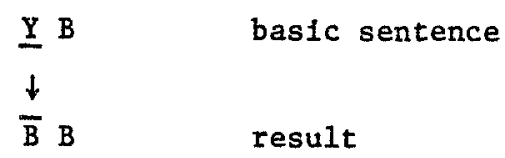

No matter what other queries are constructed for L2, the response should be red, because L2 is a finite two-sentence language containing only the sentences YB and BB. Notice that L2 contains exactly the same two sentences as L1 contains, and no others. This means that L2 is really the same language as $\mathrm{L} 1$; they are merely different formulations of the same finite language, which contains only the two sentences YB and BB. Thus we see that the same language may be formulated in different ways.

Notice that each of these first two languages can be formulated on the language mat by the use of only four chips and only two different colors of chips. Both of these languages could be built by a native in what is called a 4-2 level game of QUERIES 'N THEORIES. The pair of numbers in a 4-2 level game signifies that the native should use at most four chips and at most two colors of chips in formulating his language. 
$:$ :

: signifies the maximum number of colors of chips that native can

: use in formulating his language

signifies the maximum number of chips that native can use in formulating his language.

The first number indicates the maximum number of chips that can be used in formulating the language, and the second number indicates the maximum number of colors of chips that can be used.

Now, let us consider a more interesting language that can be built in a 4-2 level game, language L3.

L3

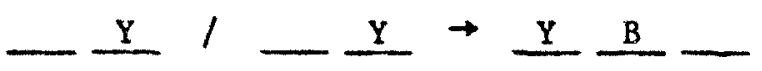

What are the appropriate responses to the following series of queries?

L3

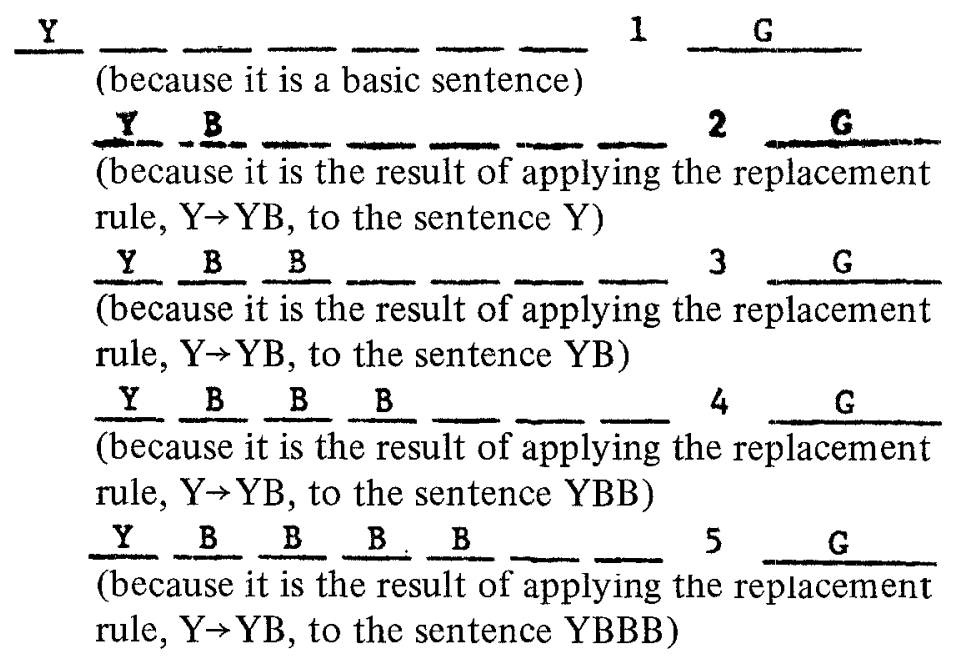

How many sentences are there in language L3? (an infinite number). How would you characterize which strings of chips are sentences in L3 and which strings are not? (A string of chips is a sentence in L3 if and only if it begins with a yellow chip which is followed by zero or more blue chips.)

Is there a longest sentence in L3? (no)

How long may a string of chips be and still be a sentence in L3? (of any length.)

Hence, at even the relatively simple 4-2 level of game in QUERIES ' $N$ THEORIES it is possible to generate interesting languages which contain an infinite number of sentences and in which sentences may be of any length. 
Now, we are ready to move up a game level and consider a language (L4) that could be built in a 5-2 level game. Up to five chips and up to two colors of chips may be used by the native in building a language on the language mat.

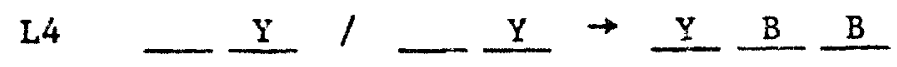

What are the appropriate responses with respect to L4 to the same five queries that were addressed to L3?

L4

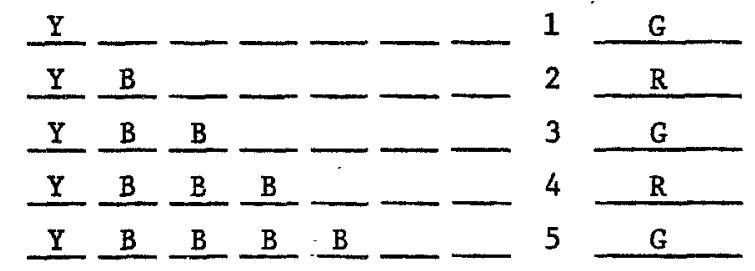

The proofs of the affirmative responses to $\# 1, \# 3$, and $\# 5$ are that (1) $\mathrm{Y}$ is a basic sentence, (3) YBB is the result of applying the replacement rule, $Y \rightarrow Y B B$, to the basic sentence, $Y$, and (5) YBBBB is the result of applying the replacement, $\mathrm{Y} \rightarrow \mathrm{YBB}$, to the sentence $\mathrm{YBB}$.

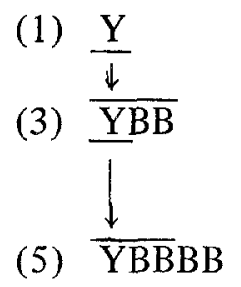

basic sentence

result of applying $\mathrm{Y} \rightarrow \mathrm{YBB}$ to the sentence $\mathrm{Y}$

result of applying $\mathrm{Y} \rightarrow \mathrm{YBB}$ to the sentence YBB

How many sentences are there in language L4? (an infinite number) Is there a longest sentence in L4? (no)

How long may a string of chips be and still be a sentence in L4? (of any length as long as the string contains an odd number of chips). How would you characterize which strings of chips are sentences in L4 and which strings are not? (A string of chips is a sentence in L4 if and only if it begins with a yellow chip which is followed by zero or an even number of blue chips.)

What is the relationship between language L3 and language L4? (L4 is included in L3; every sentence of L4 is a sentence of L3, but not vice-versa.)

With these four examples of languages in mind it is now more meaningful to answer the question: What is a language for purposes of QUERIES 'N THEORIES? In QUERIES 'N THEORIES a language is a set of sentences consisting of one or more basic sentences and zero or more generated sentences. A basic sentence of a language is a string of one or 
two colored chips that appears as a basic sentence in the formulation of the language. A generated sentence of a language is the result of applying to a sentence of the language one of the replacement rules that appears in the formulation of the language. In QUERIES 'N THEORIES a language is formulated by the native by placing colored chips on the language mat to specify the basic sentences of the language and the replacement rules of the language.

In the play of QUERIES 'N THEORIES the native secretly formulates a language on the language mat, and the querists (there may be more than one) seek to achieve an understanding of the native's language by asking him questions about it that he must answer. Querists ask questions by constructing queries on the query mat. They seek to achieve an understanding of the language such that when the native in turn asks them about it by constructing queries on the query mat, they will be able to answer correctly. Querists seek to achieve this understanding by asking the fewest numbert of questions possible, because asking questions costs.

We are now in a position to illustrate the use of the DOT, DOT, DOT, ... approach to coping with the kinds of problems that querists and the native are confronted with in the play of QUERIES ' $N$ THEORIES. Suppose that the players are engaged in a 4-2 level game and the native has formulated a language on the language mat. Consider the problem that a querist is faced with. By observing carefully (the $\mathrm{O}$ phase of the first DOT) and considering the implications of what he observes, a querist can correctly impose some strong constraints upon his theorizing when he reaches that phase of coping with the problem. Observing that they are playing a $4-2$ level game and considering the implications of that fact allows a querist to infer (1) that the native has used either one chip, or two chips, or three chips, or four chips in formulating his language and (2) that the set of chips used are either all blue, all yellow, or a mixture of the two. Observing the layout of the language mat and considering the implications of that layout along with the fact that the level of game being played is 4-2, he can correctly infer (3) that the basic sentences are either one chip long or two chips long, (4) that because there are only two blanks to the left of the arrow and three blanks to the right and there is a game rule that all replacement rules must have at least as many chips to the right of the arrow as are to the left of the arrow, the only kinds of replacement rules that can be built on the language mat are the following: $\longrightarrow \rightarrow$-or $\rightarrow-\longrightarrow$ - or $\rightarrow-\rightarrow-$ or $\rightarrow-\rightarrow--$ or $-\longrightarrow \rightarrow$ - _ - and (5) that because at least one chip must be used to build a basic sentence, there are at most only three chips left to build replacement rules and so the only kinds of replacement rules possible in this 4-2 game are $\longrightarrow \rightarrow$ and $\longrightarrow \rightarrow \ldots$ and there is at most only one of these. 
In moving to the next phase, the $\mathrm{T}$ (or theorizing) phase of coping with this problem of seeking to achieve an understanding of the native's language, it is useful to note the useful interaction between the preceeding $O$ phase and the thinking in the theorizing phase. A useful boundary is drawn around what theorizing is useful to do by the inferences from the observations in the $O$ phase. The presence of such a limiting boundary is especially useful if the querist is employing strong inference in the manner that Platt counsels - with multiple working hypotheses to explain the data. The virtues of multiple working hypotheses will certainly become convincingly apparent to most experienced players of QUERIES ' $N$ THEORIES. The argument for proceeding with multiple working hypotheses is especially strengthened when it is coupled with the limiting principle of only proceeding with hypotheses that are disconfirmable.

It is easy to agree with Platt, that these twin principles are strongly coupled to rapid scientific progress. Essentially, progress is achieved in this manner by conceiving of likely explanations of the phenomena you are interested in understanding and then designing experiments that will disprove one or more of those explanations. (The experiments sometimes also produce data that motivate researchers to conceive of additional explanations.) You advance to the good theory by disproving the bad ones. And that's really how science makes progress! (Popper, 1959) What's more, the fact that science never really proves anything in the sense of completely confirming one theory as totally sound, is part of what keeps it exciting and adventuresome. A helpful reminder of the nature of this process is embodied in a pair of severe but useful private questions that Platt suggests one ask (oneself):

on hearing any scientific explanation or theory put forward,

"But sir, what experiment could disprove your hypothesis?",

or

on hearing a scientific experiment described,

"But sir, what hypothesis does your experiment disprove?

Polya also makes the same point in memorable language (Polya, 1954):

Nature may answer Yes or No, but it whispers one answer and thunders the other, its Yes is provisional, its No is definitive.

But limiting the multiple working hypotheses to those that are disconfirmable is not the only means of keeping multiple hypotheses within manageable limits; the implications of careful prior observations are also helpful in this respect. Multiple hypotheses are useful. But how many is multiple, or, perhaps more precisely, how multiple is most useful? Armed with inferences (1)-(5), a querist can easily ascertain that there are exactly ten different disconfirmable theories - and no more to be explored in the questioning of the native. There are six possible different theories of languages consisting of various combinations of basic 
sentences alone and four different theories of languages that are formulated by combinations of both basic sentences and replacement rules:

$\mathrm{T} 1$

$\mathrm{T} 2$

T3

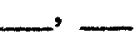

$\mathrm{T} 4$

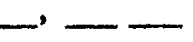

T5

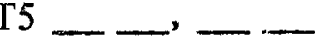

T6
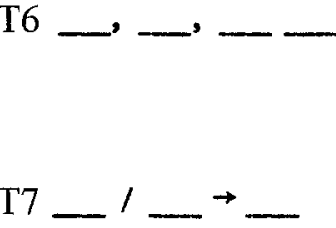

T8

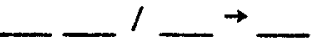

T9 __ _ _ _ _ two basic sentences (a pair of singletons) and a

T9 _- _ ' - - two basic sentences (a pair of singletons) and a

Theory that native's language consists of:

one basic sentence (a singleton) and no replacement rules

Possibilities: (Y) or (B)

one basic sentence (a doubleton) and no replacement rules

Possibilities: (BB) or (BY) or (YB) or (YY)

two basic sentences (a pair of singletons) and no replacement rules

Possibility: (Y, B)

two basic sentences (a singleton and a doubleton) and no replacement rules

Possibilities: $(\mathrm{B}, \mathrm{BB})$ or $(\mathrm{B}, \mathrm{BY})$ or $(\mathrm{B}, \mathrm{YB})$ or $(\mathrm{B}, \mathrm{YY})$ or $(\mathrm{Y}, \mathrm{BB})$ or $(\mathrm{Y}, \mathrm{BY})$ or $(\mathrm{Y}, \mathrm{YB})$ or $(\mathrm{Y}$, YY)

two basic sentences (a pair of doubletons) and no replacement rules

Possibilities: (BB, BY) or (BB, YB) or (BB, YY) or $(\mathrm{BY}, \mathrm{YB})$ or $(\mathrm{BY}, \mathrm{YY})$ or $(\mathrm{YB}, \mathrm{YY})$

three basic sentences (a pair of singletons and a doubleton)

Possibilities: $(\mathrm{B}, \mathrm{Y}, \mathrm{BB})$ or $(\mathrm{B}, \mathrm{Y}, \mathrm{BY})$ or $(\mathrm{B}, \mathrm{Y}$, $\mathrm{YB})$ or $(\mathrm{B}, \mathrm{Y}, \mathrm{YY})$

one basic sentence and a replacement rule with a singleton changing to a singleton

Possibilities: $(\mathrm{B} / \mathrm{B} \rightarrow \mathrm{Y})$ or $(\mathrm{Y} / \mathrm{Y} \rightarrow \mathrm{B})$

one basic sentence (a doubleton) and a replacement rule with a singleton changing to a singleton

Possibilities:

$(\mathrm{BB} / \mathrm{B} \rightarrow \mathrm{Y})$ or $(\mathrm{BY} / \mathrm{B} \rightarrow \mathrm{Y})$ or

$(\mathrm{BY} / \mathrm{Y} \rightarrow \mathrm{B})$ or $(\mathrm{YB} / \mathrm{B} \rightarrow \mathrm{Y})$ or

$(\mathrm{YB} / \mathrm{Y} \rightarrow \mathrm{B})$ or $(\mathrm{YY} / \mathrm{Y} \rightarrow \mathrm{B})$

(Note that the other possible rules do not generate any new results.) replacement rule with a singleton changing to a singleton

Possibilities: B, Y / $\rightarrow-$

(Note that no rule generates any new results.) 
_ $\quad \rightarrow$ _ one basic sentence (a singleton) and a replacement rule with a singleton changing to a doubleton

Possibilities:

$$
\begin{aligned}
& (B / B \rightarrow B B) \text { or }(B / B \rightarrow B Y) \text { or } \\
& (B / B \rightarrow Y B) \text { or }(B / B \rightarrow Y Y) \text { or } \\
& (Y / Y \rightarrow B B) \text { or }(Y / Y \rightarrow B Y) \text { or } \\
& (Y / Y \rightarrow Y B) \text { or }(Y / Y \rightarrow Y Y)
\end{aligned}
$$

In moving to the $\mathrm{D}$ or design phase (the second DOT) a querist should build a query that will disconfirm one or more of the ten theories, no matter what the outcome is, just as the scientist should design an experiment that will disconfirm one or more of his multiple working hypotheses, no matter how the experiment comes out.

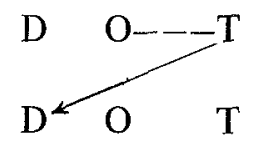

Consider the effects of building a query consisting of a single blue chip alone. If the outcome is green, then theories T2, T5, and T8 are disconfirmed; if the outcome is red, then theories T3, T6, T7, and T9, are disconfirmed. Therefore, the query is a good one to reduce the number of possible theories. Suppose that that outcome is green:

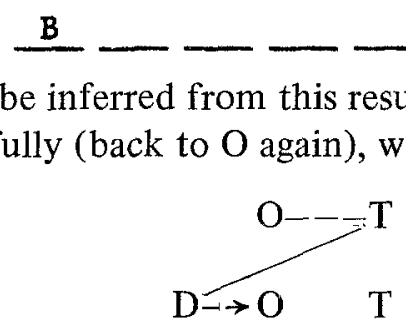

No, it cannot be inferred that $B$ is a basic sentence, because it might be a generated sentence by virtue of $\mathrm{T} 7$. All that can be inferred (back to $\mathrm{T}$ again) is that $\mathrm{T} 2, \mathrm{~T} 5$, and $\mathrm{T} 8$ are disconfirmed.

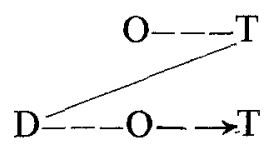

What is a good question to ask next (back to $\mathrm{D}$ again) to disconfirm one or more of the remaining seven theories. 


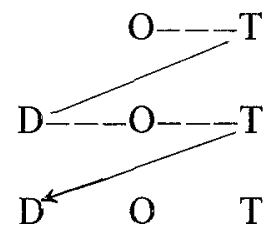

Consider the effects of building a query consisting of a single yellow chip alone as the second query in the context of what is already known from the results of the first query. If the outcome is green, then theories $\mathrm{T} 1, \mathrm{~T} 4$, and T1 0 are disconfirmed; if the outcome is red, then theories T3, T6, T7, and T9 are disconfirmed. Therefore, the query is a good one to reduce the possibilities. Suppose that the outcome is red:

$$
\underline{\mathrm{Y}}-\longrightarrow-\longrightarrow \mathrm{R}
$$

Observe (back to $\mathrm{O}$ again), what can the querist infer from this result?

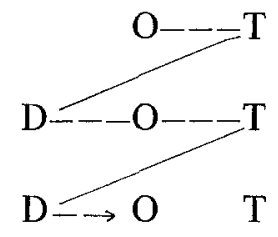

He now knows (back to $\mathrm{T}$ again) that $\mathrm{T} 3, \mathrm{~T} 6, \mathrm{~T} 7$, and $\mathrm{T} 9$ are disconfirmed and that $\mathrm{B}$ is a basic sentence, because none of the only three theories that remain as possible explanations of the data generated so far (T1, T4, and $\mathrm{T} 10)$ can have B as a generated sentence; so B must be a basic sentence.

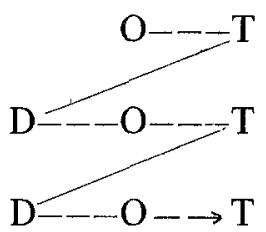

What is a good question to ask next (back to D again) to disconfirm one or more of the remaining three theories?

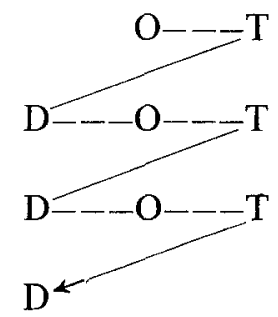

Consider the effects of building $\mathrm{BB}$ as the third query in the context 
of what is already known from the results of the first two queries.

If the outcome is green, then T1 is disconfirmed; but if the outcome is red then none of the three remaining theories are disconfirmed. Therefore, it is not a query that will definitely reduce the possibilities. Is there any such query in the circumstances? Notice that the queries BY, YB and YY all have the same limitations: a red outcome does not disconfirm any of the three remaining theories. In some circumstances there does not exist any query (of the type that has been considered so far) that will guarantee, no matter what the outcome to the query is, disconfirmation of at least one theory. Sometimes the best that can be accomplished by a query regardless of outcome is to reduce the number of queries that may have to be built before reaching one that will definitely disconfirm at least one theory regardless of outcome. This is one example of such circumstances. Each of the four queries above does disconfirm T1 if the outcome is green, but none of them disconfirms any of the three remaining theories if the outcome is red. And there is no other query of this type that will guarantee disconfirmation of at least one theory regardless of the outcome.

Suppose that the query built in fact is BB and that the outcome is green:

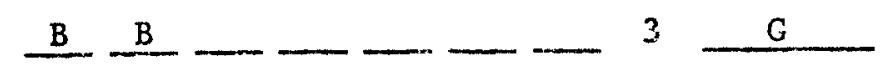

Observe (back to $O$ again), what can the querist infer from this result? He now knows (back to $T$ again) that $T 1$ is disconfirmed and that the native's language is either $(\mathrm{B}, \mathrm{BB})$ of $\mathrm{T} 4$ or $(\mathrm{B} / \mathrm{B} \rightarrow \mathrm{BB})$ of $\mathrm{T} 10$.

The querist is now ready for a crucial experiment. What is a good question to ask next (back to D again) that will disconfirm one of the two remaining explanations of what the native's language is? Consider the effects of building BBB as the fourth query in the context of what is already known from the results of the first three queries. If the outcome is green, then T4 is disconfirmed; if the outcome is red, then T10 is disconfirmed. Therefore, it is a good query (it is the equivalent of a crucial experiment) to determine the language.

Whatever the outcome (suppose it is green), the querist will understand the native's language. When he feels confident that he understands the native's language well enough to answer correctly any question that the native can construct on the query mat, the querist should declare, "Aha," signifying his claim that he can answer all of the native's questions correctly. If the querist fails to answer all of the native's questions correctly, then the querist loses. If he does answer all of the questions correctly, then he wins, provided that the number of questions that he has asked in learning the native's language does not exceed some maximum 
specified by the game rules. In detecting the native's language in this 4-2 level game the querist required only four queries to determine that it was $(\mathrm{B} / \mathrm{B} \rightarrow \mathrm{BB})$. In doing so he went through exactly four complete DOT cycles (that is, OT, DOT, DOT, DOT, DOT). Even in this relatively simple example, the sharp convergence obtained by reducing the number of theories compatible with the data through the repeated application of strong inference technique is apparent. In more complex examples, with more possibilities, the necessity for such convergence is even more apparent, and players are unlikely to miss the usefulness of strong inference methods to achieve it.

However, while the convergence necessary is affected by the complexity of language possible at the game level being played, its availability is influenced by the generality of questioning permitted by the game rules. At the very next game level it becomes apparent that the kind of questioning considered so far is extremely weak in detecting languages permissible at the 5-2 level - extremely weak in the sense that it may require many questions to detect the language with certainty. Suppose that the native formulated language L5 on the language mat:

\section{L5 B / B $\rightarrow$ YYY}

Although the querist may be asking as good a question as it is possible for him to ask on the basis of the information that he has available at each stage of the play, he may require 14 queries to generate enough data to understand the native's language with certainty if the questioning follows this sequence:

LS
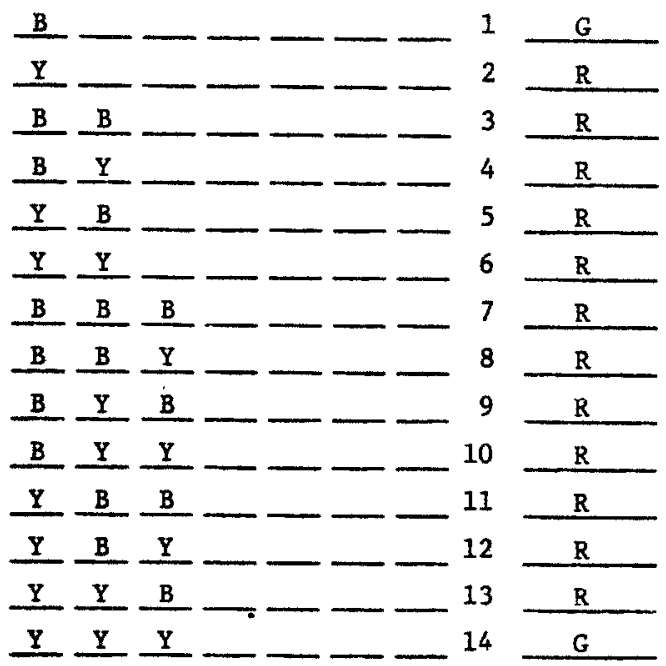

When limited to asking specific questions of the sort, "Is this string of chips a sentence in the language?" the querist will in some cases obtain 
very little data to narrow down the range of possible languages even though he is proceeding systematically and posing perfectly good queries on the basis of what information is available. This points up the need for game rules which will allow the querist to ask more general questions; for example, of the type, "Is there any sentence in the language that has this property?" Permitting this more general type of questioning is accomplished in QUERIES 'N THEORIES by introducing the use of the white chip to construct what are called "strong queries." A white chip stands for a string of chips (possibly empty) of unspecified length with colors of chips unspecified; it indicates an elision, very much like the use of three dots (. . ) in ordinary prose. Every empty space in a strong query, except those that precede an initial white chip or follow a terminal white chip, stands for a single chip of unspecified color. Consider what can be done with the availability of white chips for constructing strong queries.

$$
\underline{\mathrm{Y}} \mathrm{W}-\longrightarrow-15
$$

This query asks the question:

Is there any sentence in the language that has the property of beginning with a yellow chip?

More briefly:

$$
\mathrm{Y} \ldots \text { ? }
$$

On the other hand,

$$
-1-16
$$

asks the question, "Is there any sentence in the language that has the property of ending with a yellow chip?"; that is, ". . Y Y?" More than one white chip can be used in a strong query,

$$
\underline{\mathrm{W}} \mathrm{W}-17
$$

to ask, "Is there any sentence in the language that contains a yellow chip?"; that is, ". . . Y . . ?" Blank spaces can also be used to advantage. (Recall that only those blanks preceding initial white chips or following terminal white chips are ignored.) The following strong query,

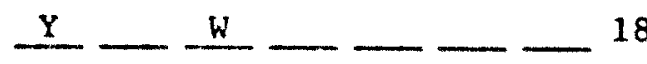

asks, "Is there any sentence in the language at least two chips long that begins with a yellow chip?"; that is, "Y_...?" The following,

asks, "Is there any sentence in the language at least two chips long that ends with a blue chip?"; that is, ".....B?" 
The availability of strong queries as well as specific queries permits a much more rapid convergence in narrowing the range of possible languages in a 5-2 (or higher) level of game. With the inferences from the responses in the following sequence of queries one can detect language L5 with just seven questions:

Queries

L5

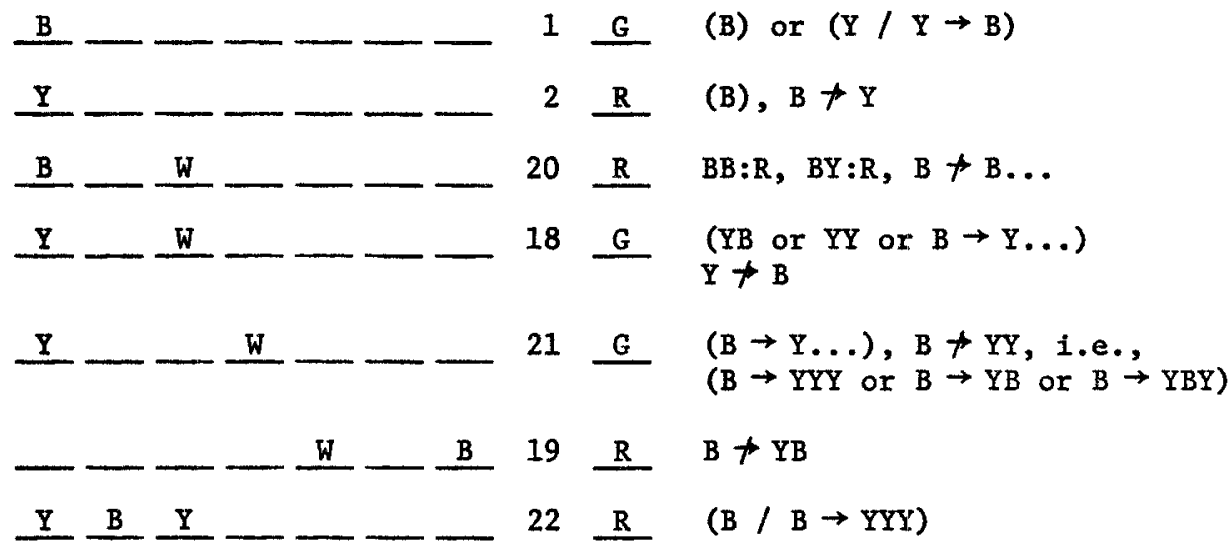

Outcomes Inferences

Notice that in the context of the responses to queries 1 and 2 the response to query 20 provides all the data that was provided by the responses to queries $3,4,7,8,9$, and 10 . The green response to query 18 indicates that there is a sentence in the language that begins with a yellow chip, which must be so by its being a basic sentence or being generated by a rule. The green response to query 21 indicates that the sentence beginning with a yellow chip is generated by means of a rule - one of the following three: $B \rightarrow Y B, B \rightarrow Y B Y$, or $B \rightarrow Y Y Y$. The red responses to queries 19 and 22 indicate that the rule is neither $B \rightarrow Y B$ nor $B \rightarrow Y B Y$; so it must be $B \rightarrow Y Y Y$. Hence, the language is: $B / B \rightarrow Y Y Y$.

By the time players reach the 6-2 level, the natives can build languages with multiple rules and languages that are universal or nearly universal. Consider, for example, language L6. It is a 6-2 universal language; every possible string of yellow or blue chips, or combinations of them, are sentences in L6.

$$
\text { L6 } \quad B / \underset{a}{B} \rightarrow B B, \underset{b}{B} \rightarrow Y
$$

(The ' $a$ ' and the ' $b$ ' under $B \rightarrow B B$ and $B \rightarrow Y$ are merely to indicate the names of the rules so that they can easily be referred to.) For example, BBYBYYB can be generated from the basic sentence in L6 by applying the rules in the following order: $a$ a $a$ a $a \mathrm{a} b \mathrm{~b}$ b. Language $\mathrm{L} 7$ is almost $\mathrm{a}$ 6-2 level universal language. Every possible string of yellow or blue, except one, is a sentence. 
(Can you spot which string is not a sentence?)

By the time the players reach game level $7-3$, one can presume that they have achieved insight into the usefulness of first determining the basic sentences of the unknown language. To avoid the necessity of beginning each play of the game by constructing all the possible specific singleton and doubleton queries, the native is required to reveal all those singletons and doubletons that are sentences in his language Any not revealed to be sentences must not be sentences. If they are, the native will ultimately suffer heavily in the scoring.

It pays to think about what can be inferred from the revelations. If the native reveals in an 8-3 level game that B (blue), $P$ (purple), $Y$ (yellow), $\mathrm{BB}, \mathrm{BP}, \mathrm{PB}, \mathrm{PP}, \mathrm{YB}$, and $\mathrm{YP}$ are sentences in his language (and implicitly that BY, PY, and YY are not), he has provided enough data that the language can be determined merely by asking two simple questions. By observing carefully, theorizing imaginatively, and designing rigorously, a querist can ascertain that there are exactly three languages possible in an 8-3 level game that are compatible with the native's revelations. By constructing the pair of specific queries YBB and YYB, he can determine which of the three is the native's language no matter how the native responds to the queries. Why is this so? The DOT, DOT, DOT, . . analysis goes like this.

If there are three singletons in the native's language, then they must be obtained in one of the following three ways:

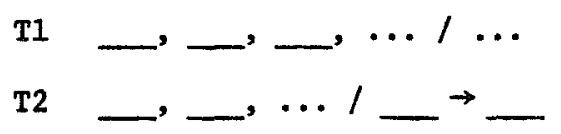

T3

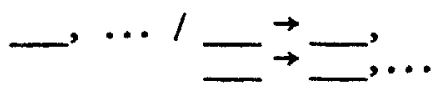

(three singleton basic sentences)

(two singleton basic sentences and a 1-to-1 rule)

(a singleton basic sentence and two 1-to-1 rules)

If the three singletons are by virtue of $\mathrm{T} 1$, then there are just five chips left to get the six doubletons of the native's language.

The five chips might be used in any one of the following four ways:

T1.1

T1.2

T1.3

T1.4

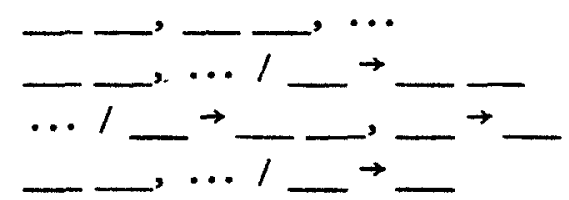

But in any of the languages formulated by T1.1 or T1.2 there will only be two doubletons, rather than the six revealed by the native. Hence, $\mathrm{T} 1.1$ and $\mathrm{T} 1.2$ are disconfirmed by the data. At most, four doubletons can 
occur in any of the languages formulated by T1.3 or T1.4 (for example, $\mathrm{BB}, \mathrm{BP}, \mathrm{PB}$, and $\mathrm{PP}$ are sentences in the language $\mathrm{B}, \mathrm{P}, \mathrm{Y} / \mathrm{P} \rightarrow \mathrm{PP}, \mathrm{P} \rightarrow \mathrm{B})$; so, T1.3 and T1.4 are also disconfirmed. Since any other usage of the five remaining chips than in one of the above four ways will generate even less doubletons, $\mathrm{T} 1$ is disconfirmed.

If the three singletons in the language are by virtue of $\mathrm{T} 2$, there are only nine possible theories that have just a pair of singleton basic sentences:

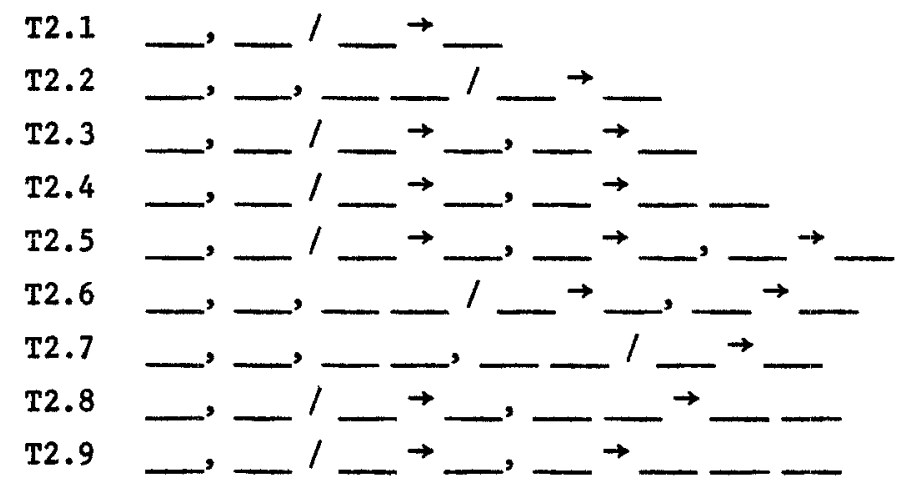

However, there are no doubletons in any of the languages formulated by $\mathrm{T} 2.1, \mathrm{~T} 2.3, \mathrm{~T} 2.5, \mathrm{~T} 2.8$, or $\mathrm{T} 2.9$. There are, at most, four doubletons in any languages formulated by $\mathrm{T} 2.2$ or $\mathrm{T} 2.4$ (for example, $\mathrm{Y}, \mathrm{B}, \mathrm{BB} / \mathrm{B} \rightarrow \mathrm{P}$ ). This disconfirms each of them leaving only $\mathrm{T} 2.6$ and $\mathrm{T} 2.7$ as possibilities.

Since $Y$ is revealed to be a sentence in the Native's language, it must be a sentence by virtue of one of the three following possibilities:

$\begin{aligned} & \mathrm{T} 2.6 \mathrm{a} \\ & \mathrm{T} 2.6 \mathrm{~b}\end{aligned} \ldots / \mathrm{B} \rightarrow \mathrm{Y}, \mathrm{Y}, \mathrm{Y}, \ldots$

T2.6c Y, ... / ..

Since $Y B$ is a sentence in the native's language and $Y Y$ is not, there cannot be a $B \rightarrow Y$ rule in the formulation of the native's language. Similarly, since YP is a sentence in the native's language and YY is not, there cannot be a $\mathrm{P} \rightarrow \mathrm{Y}$ rule in the formulation of the native's language. Hence, T2.6a and T2.6b are disconfirmed.

The doubleton in $\mathrm{T} 2.6 \mathrm{c}$ must begin with either $\mathrm{B}, \mathrm{P}$, or $\mathrm{Y}$; hence, there are just the three following alternatives:

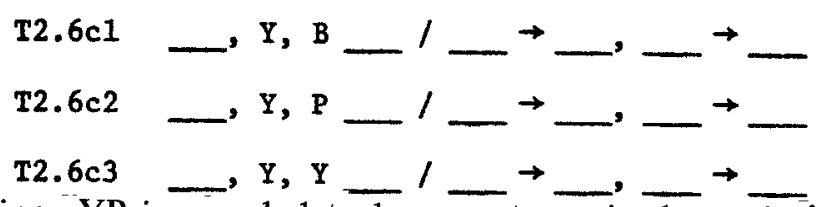

Since YP is revealed to be a sentence in the native's language, and in $\mathrm{T} 2.6 \mathrm{cl} \mathrm{B}-$ is the only doubleton that is a basic sentence, YP must be 
directly or indirectly generated from $B$ by either $B \rightarrow Y$ or by the pair of rules, $\mathrm{B} \rightarrow \mathrm{P}$ and $\mathrm{P} \rightarrow \mathrm{Y}$. However, it has already been shown above that neither $\mathrm{B} \rightarrow \mathrm{Y}$ nor $\mathrm{P} \rightarrow \mathrm{Y}$ can be in the formulation of the language; hence, $\mathrm{T} 2.6 \mathrm{cl}$ is disconfirmed. Similarly, T2.6c2 is disconfirmed, leaving only $\mathrm{T} 2.6 \mathrm{c} 3$.

The other singleton in $\mathrm{T} 2.6 \mathrm{c} 3$ is either $\mathrm{B}, \mathrm{P}$, or $\mathrm{Y}$; thus, there are three possibilities:

$\mathrm{T} 2.6 \mathrm{c} 3 \mathrm{~A} \quad \mathrm{~B}, \mathrm{Y}, \mathrm{Y} \_\prime+\longrightarrow$

$\mathrm{T} 2.6 \mathrm{c} 3 \mathrm{~B} \quad \mathrm{P}, \mathrm{Y}, \mathrm{Y} \_$_ $\longrightarrow \longrightarrow$

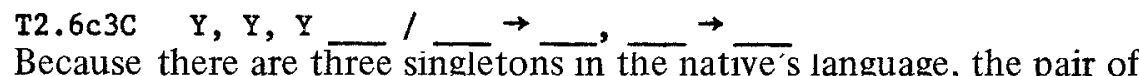
1-to-1 rules of $\mathrm{T} 2.6 \mathrm{c} 3 \mathrm{C}$ must be $\mathrm{Y} \rightarrow \mathrm{B}$ and $\mathrm{Y} \rightarrow \mathrm{P}$. However, if that is so, the maximum number of doubletons there can be in any language formulated by $\mathrm{T} 2.6 \mathrm{c} 3 \mathrm{C}$ is three, because YY cannot be the basic sentence since it is not a sentence of the native's language. Since the native's language has six doubletons, $\mathrm{T} 2.6 \mathrm{c} 3 \mathrm{C}$ is disconfirmed.

Because $\mathrm{YY}$ is not a sentence in the native's language, the second chip in the doubleton of $\mathrm{T} 2.6 \mathrm{c} 3 \mathrm{~A}$ and of $\mathrm{T} 2.6 \mathrm{c} 3 \mathrm{~B}$ must be either $\mathrm{B}$ or $\mathrm{P}$; thus, there are four possibilities:

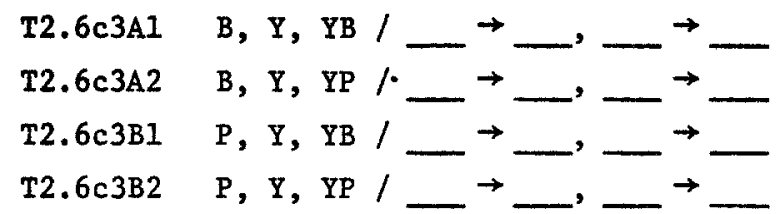

In order to generate the singletons and doubletons revealed to be sentences of the native's language (i.e., B, P, Y, BB, BP, PB, PP, YB, and $\mathrm{YP}$ ), the pair of rules to $\mathrm{T} 2.6 \mathrm{c} 3 \mathrm{~A} 1$ and $\mathrm{T} 2.6 \mathrm{c} 3 \mathrm{~B} 1$ must be $\mathrm{Y} \rightarrow \mathrm{B}$ and $\mathrm{B} \rightarrow \mathrm{P}$ and the pair of rules to $\mathrm{T} 2.6 \mathrm{c} 3 \mathrm{~A} 2$ and $\mathrm{T} 2.6 \mathrm{c} 3 \mathrm{~B} 2$ must be $\mathrm{Y} \rightarrow \mathrm{P}$ and $\mathrm{B} \rightarrow \mathrm{B}$. This results in the following four formulations of a language:

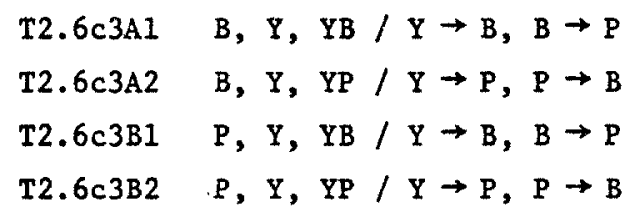

Each of these four alternatives formulates exactly the same language.

It is the language that contains just the revealed singletons and doubletons of the native's language; it does not have any sentences containing three or more chips. Hence, the language formulated by these four $\mathrm{T} 2.6 \mathrm{c} 3$ formulations is compatible with the data revealed, and these four alone among the T2.6 formulations result in a language compatible with the data. 
Similarly, the following pair of T2.7 formulations are the only ones compatible with the three singletons and six doubletons revealed to be sentences in the native's language:

T2.7a B, Y, BB, YB / B $\rightarrow$ P

T2.7b P, Y, PP, YP / P $\rightarrow$ B

These two T2.7 alternatives formulate exactly the same language as that formulated by the four T2.6c3 alternatives above. These six formulations are the only $\mathrm{T} 2$ formulations that are compatible with the native's revelations; they all formulate the same language, one which has as its only sentences those relevations.

Turning now to the T3 formulations, there are only four possibilities having just one singleton basic sentence.

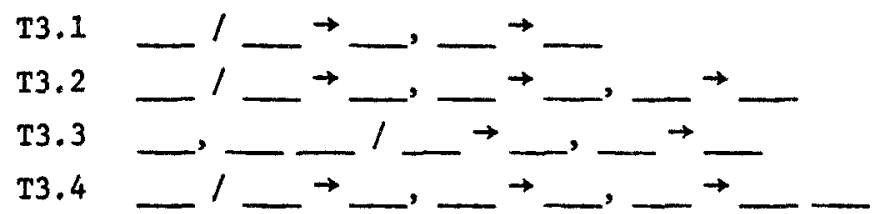

Since there are no doubletons in the languages formulated by T3.1 or T3.2, they are both disconfirmed.

There are two T3.3 formulations that are compatible with the three singleton and six doubleton revelations of the native's language, and they are the only compatible ones.

$$
\begin{aligned}
& \text { T3.3a } \mathrm{Y}, \mathrm{YB} / \mathrm{Y} \rightarrow \mathrm{B}, \mathrm{B} \rightarrow \mathrm{P} \\
& \mathrm{T} 3.3 \mathrm{~b} \\
& \mathrm{Y}, \mathrm{YP} / \mathrm{Y} \rightarrow \mathrm{P}, \mathrm{P} \rightarrow \mathrm{B}
\end{aligned}
$$

This pair of T3.3 alternatives formulates the same language that is formulated by the six $\mathrm{T} 2$ formulations that were compatible with the revelations.

This leaves one T3 theory to be considered, those formulations of the type indicated in T3.4. In considering T2.6, it was shown that neither $\mathrm{B} \rightarrow \mathrm{Y}$ nor $\mathrm{P} \rightarrow \mathrm{Y}$ could be part of a formulation of a language compatible with the revealed data. Since there cannot be any rules that will generate a singleton $\mathrm{Y}$ from some other singleton, the singleton basic sentence in T3.4 must be $\mathrm{Y}$. With $\mathrm{Y}$ as the singleton basic sentence, there are just three ways for the other singletons to be generated by a pair of 1-to-1 rules.

$$
\begin{aligned}
& \text { T3.4a } \quad Y / Y \rightarrow B, Y \rightarrow P, \ldots \\
& \text { Y3.4b } \quad Y / Y \rightarrow B, B \rightarrow P, \ldots \\
& \text { T3.4C } \quad Y / Y \rightarrow P, P \rightarrow B, \ldots
\end{aligned}
$$


There are 27 different 1-to- 2 rules to be considered as the other rule in T3.4a, but each of the 27 languages so formulated fails to match the revelations in some respect. Each of the languages formulated by adding $\mathrm{B} \rightarrow \mathrm{YY}, \mathrm{P} \rightarrow \mathrm{YY}$, or $\mathrm{Y} \rightarrow \mathrm{YY}$ generates the doubleton $\mathrm{YY}$ as a sentence, but it is not a sentence of the native's language according to the revealed data. Each of the languages formulated by adding $B \rightarrow Y P, P \rightarrow Y P$, or $Y \rightarrow Y P$ fails to generate the doubleton $\mathrm{PB}$ as a sentence, and it is one of the revealed sentences. Each of the languages formulated by adding each of the other 21 possible rules fails to generate the doubleton $\mathrm{YP}$ as a sentence, and it is one of the revealed sentences. Thus, T3.4a is disconfirmed, leaving only $\mathrm{T} 3.4 \mathrm{~b}$ and $\mathrm{T} 3.4 \mathrm{c}$.

If $\mathrm{Y} \rightarrow \mathrm{YB}$ is the 1 -to- 2 rule of $\mathrm{T} 3.4 \mathrm{~b}$, then the doubletons revealed to be sentences of the native's language will all be sentences in the language thus formulated, and only those doubletons will be sentences. Only two other rules considered as the 1-to- 2 rule of $\mathrm{T} 3.4 \mathrm{~b}$ would produce these same results; these rules are $\mathrm{B} \rightarrow \mathrm{YB}$ and $\mathrm{P} \rightarrow \mathrm{YB}$. All other possible rules considered as the 1-to- 2 rule of $\mathrm{T} 3.4 \mathrm{~b}$ formulate languages that either do not have all the revealed doubletons as sentences or have as sentences some doubletons that are not revealed as sentences.

Therefore, these three are all the possible $\mathrm{T} 3.4 \mathrm{~b}$ alternatives:

$$
\begin{aligned}
& \text { T3.4b1 } \mathrm{Y} / \mathrm{Y} \rightarrow \mathrm{B}, \mathrm{B} \rightarrow \mathrm{P}, \mathrm{Y} \rightarrow \mathrm{YB} \\
& \mathrm{T} 3.4 \mathrm{~b} 2 \\
& \mathrm{Y} / \mathrm{Y} \rightarrow \mathrm{B}, \mathrm{B} \rightarrow \mathrm{P}, \mathrm{B} \rightarrow \mathrm{YB} \\
& \mathrm{T} 3.4 \mathrm{~b} 3 \quad \mathrm{Y} / \mathrm{Y} \rightarrow \mathrm{B}, \mathrm{B} \rightarrow \mathrm{P}, \mathrm{P} \rightarrow \mathrm{YB}
\end{aligned}
$$

In the language formulated by $\mathrm{T} 3.4 \mathrm{~b} 1$ the following three-chip strings are sentences:

$\begin{array}{lll}\text { BBB } & \text { PBB } & \text { YBB } \\ \text { BBP } & \text { PBP } & \text { YBP } \\ \text { BPB } & \text { PPB } & \text { YPB } \\ \text { BPP } & \text { PPP } & \text { YPP }\end{array}$

In general, any string of chips that has as its first chip a blue, a purple, or a yellow chip which is followed by any combination of blue or purple chips is a sentence in the language formulated by $\mathrm{T} 3.4 \mathrm{~b} 1$.

The language formulated by $\mathrm{T} 3.4 \mathrm{~b} 2$ and the language formulated by $\mathrm{T} 3.4 \mathrm{~b} 3$ have as sentences all those that are sentences of $\mathrm{T} 3.4 \mathrm{~b} 1$ plus some additional ones. Both formulate a language that has exactly the same additional sentences, so they both formulate the same language. The following three-chip strings, in addition to those in the language formulated by $\mathrm{T} 3.4 \mathrm{~b} 1$, are sentences in the language formulated by $\mathrm{T} 3.4 \mathrm{~b} 2$ and by T3.4b3. 


\section{BYB PYB YYB \\ BYP PYP YYP}

In general, any string of chips that is two or more chips long, that is comprised of any combination of blue, purple, or yellow chips, and that has as its last chip a blue or purple chip - any such string is a sentence in the language formulated by $\mathrm{T} 3.4 \mathrm{~b} 2$ and by $\mathrm{T} 3.4 \mathrm{~b} 3$.

Turning now to the languages formulated by $\mathrm{T} 3.4 \mathrm{c}$, there are just three 1-to-2 rules that result in languages that have exactly the right doubletons as sentences to match those that are revealed as sentences in the native's language. They are $\mathrm{Y} \rightarrow \mathrm{YP}, \mathrm{B} \rightarrow \mathrm{YP}$, and $\mathrm{P} \rightarrow \mathrm{YP}$; and the three accompanying $\mathrm{T} 3.4 \mathrm{c}$ alternatives parallel those of $\mathrm{T} 3.4 \mathrm{~b}$ :

$$
\begin{aligned}
& \text { T3.4 C1 Y / Y } \rightarrow \text { P, P } \rightarrow \text { B, Y } \rightarrow \text { YP } \\
& \text { T3.4 } 2 \text { 2 } \mathrm{Y} / \mathrm{Y} \rightarrow \mathrm{P}, \mathrm{P} \rightarrow \mathrm{B}, \mathrm{B} \rightarrow \mathrm{YP} \\
& \text { T3.4c3 Y / Y } \rightarrow P, P \rightarrow B, P \rightarrow Y P
\end{aligned}
$$

It turns out that all the strings of chips that are sentences in the language formulated by $\mathrm{T} 3.4 \mathrm{bl}$ are also sentences in the language formulated by $\mathrm{T} 3.4 \mathrm{cl}$, and vice-versa. So T3.4b1 and T3.4c1 formulate the same language. Similarly, all the strings of chips that are sentences in the language formulated by $\mathrm{T} 3.4 \mathrm{~b} 2$ and by $\mathrm{T} 3.4 \mathrm{~b} 3$ are also sentences in the language formulated by $\mathrm{T} 3.4 \mathrm{c} 2$ and are also sentences in the language formulated by T3.4c3, and vice-versa in each case. So T3.4b2, T3.4b3, $\mathrm{T} 3.4 \mathrm{c} 2$, and $\mathrm{T} 3.4 \mathrm{c} 3$ all formulate the same language.

The analysis above covers all the possibilities, and the revealed data has narrowed the alternatives down to the following three languages:

L1 the language formulated by $\mathrm{T} 2.6 \mathrm{c} 3 \mathrm{~A} 1$, by $\mathrm{T} 2.6 \mathrm{c} 3 \mathrm{~A} 2$, by $\mathrm{T} 2.6 \mathrm{c} 3 \mathrm{~B} 1$, by $\mathrm{T} 2.6 \mathrm{c} 3 \mathrm{~B} 2$, by $\mathrm{T} 2.7 \mathrm{a}$, by $\mathrm{T} 2.7 \mathrm{~b}$, by $\mathrm{T} 3.3 \mathrm{a}$, and by $\mathrm{T} 3.3 \mathrm{~b}$

L2 the language formulated by T3.4b1 and by T3.4c1

$\mathrm{L} 3$ the language formulated by $\mathrm{T} 3.4 \mathrm{~b} 2$, by $\mathrm{T} 3.4 \mathrm{~b} 3$, by $\mathrm{T} 3.4 \mathrm{c} 2$, and by $\mathrm{T} 3.4 \mathrm{c} 3$.

Notice that the string $\mathrm{YBB}$ is a sentence in $\mathrm{L} 2$ and $\mathrm{L} 3$, but not in $\mathrm{L} 1$; and that string YYB is a sentence in L3, but not in L2. Therefore, queries about these two strings will be effective in determining the native's language regardless of how he responds to the queries. If he indicates green to the query

$$
\text { Y Y B }-1-1
$$

then both $\mathrm{L} 1$ and $\mathrm{L} 2$ are disconfirmed and the native's language is determined to be L3. On the other hand, if he indicates red, then L3 is 
disconfirmed and the second query must be posed to determine the native's language:

$$
\text { Y B } \text { B }-2-2
$$

If the response to this second query is green, then $\mathrm{L} 1$ is disconfirmed and the native's language is determined to be L2. If the response is red, then $\mathrm{L} 2$ is disconfirmed and the native's language is determined to be L1.

If it is not already apparent that players of this game have the opportunity to learn a powerful analytic skill by considering a programmed series of problems that gradually and steadily increase in complexity, and if the above 8-3 level example is not already persuasive of the high level thought that is necessary in order to play QUERIES ' $N$ THEORIES effectively and the intimate relationship of that kind of thought with the strong inference approach to scientific method, then perhaps a final example will be the ultimate clincher. The example is drawn from a 10-5 level game; the querist who did the analysis is an experienced high school player. Knowing that the five colors of chips

Table I

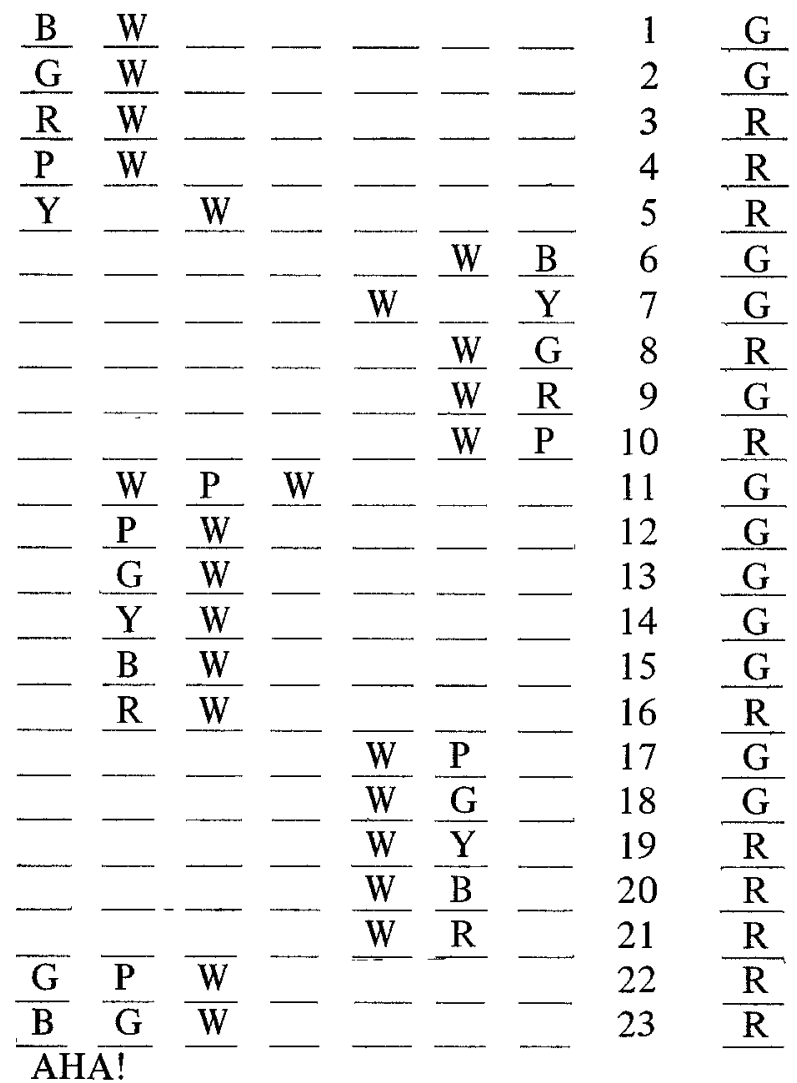


available in the game were B (blue), $\mathrm{G}$ (green), $\mathrm{P}$ (purple), R (red), and $\mathrm{Y}$ (yellow), and that the native's only revelation was $\mathrm{Y}$, he constructed the sequence of queries in Table 1.

As a test of your own skill in the use of the strong inference approach to scientific method, you might try your hand at determining from the data produced in the responses to these 23 queries what the native's language must be and why the data unerringly indicate it to be so.

As a further test, you might consider whether the language might have been determined in fewer queries, starting out the inquiry as this querist did. (Hint: With somewhat stronger theorizing after the response to query 9 , the language could have been determined with just five more queries.)

I conclude that these examples are sufficiently persuasive to indicate the value of some rigorous empirical investigation of a series of hypotheses about the merits of QUERIES 'N THEORIES as a vehicle for practicing and improving skill in the use of the strong inference approach to scientific method. That, of course, is the task ahead. My hope, and hypothesis, should be unmistakably clear: that the results will convincingly show the unjustifiability of uniformly and uncritically associating games with connotations of triviality. May QUERIES 'N THEORIES prove to be a good instructional game - one that enables players to rehearse varieties of behavior that will be useful to them in other contexts. My wildest daydream is that QUERIES 'N THEORIES will become the counterexample that leaps to mind to help resolve an important controversy: for example, when somebody is deploring the chances of bringing Lord Snow's two cultures (Snow, 1959) closer together by upgrading the understanding of science among those who do not comprehend its processes, and a strong inference thinker inquires, "But sir, what experiment could disprove your hypothesis?"

\section{References}

Allen, Layman E., Kugel, P. and Ross, Joan (1970), Queries 'N Theories: The Game of Science and Language. New Haven: Autotelic Instructional Materials Publishers.

Platt, John R. (1964). "Strong inference." Science, 144:347-353.

Polya, G. (1954), Mathematics and Plausible Reasoning. Vol. I: Induction and Analogy in Mathematics. Princeton: Princeton University Press.

Popper, K. R. (1959). The Logic of Scientific Discovery. New York: Basic Books.

Snow, C. P. (1959). The Two Cultures and the Scientific Revolution. London: Macmillan.

Von Neumann, J. and Morgenstern, O. (1944). Theory of games and economic behavior. Princeton: Princeton University Press. 